those medical men who have given it a fair trial should send you the results of their practice, to be arranged statistically afterwards, according as they have been successful or otherwise. I myself have employed it in four cases, in three of which it was eminently advantageous. In two instances, which were very severe, relapses afterwards occurred, but the patients ultimately recovered? In the fourth case, that of an old lady in collapse, the disease occurring after occasional diarrhœe for three weeks, I have reason to believe the calomel was not exhibited according to my directions; that is to say, it was not given every ten minutes. I prefer prescribing it in the form of powder, as there must be greater difficulty in swallowing a pill during the stage of collapse, or while the patient is writhing under severe general spasm, than in having a powder dropped into the mouth. Many persons cannot take a pill, even when in the enjoyment of good health; with them the taking a calomel pill every ten minutes would amount to an impossibility. I am, Sir, your obedient servant,
Tavistock-street, Covent-garden, Oct. 1849 .

** We should be glad to find the members of the profession ready to furnish reports of all the cases of cholera attended by each of them, with the results following their different methods of treatment; but we must suggest to our correspondent, that the subsequent classification of these results would be a duty far too laborious for us to attempt. The Board of Health might properly undertake the task, and employ clerks for the purpose, who ought to be young men of the medical profession not yet in practice. Their labours would not be very difficult if practitioners recorded their cases on an uniform plan. A registrar for cholera cases was published last year by Mr. Smith, of Long-acre, and noticed at

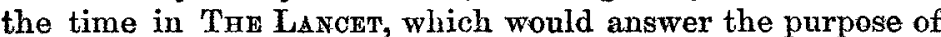
registration on an uniform method. That case-book, indeed, admits of material improvement; mearwhile, until superseded by a better and more practical form of registry, it might be used with advantage.-ED. L.

\section{TREATMENT OF CHOLERA IN INDIA.} To the Editor of The LaNCET.

Srr,-I have just received the enclosed from India. The author is unknown to me; but as he wishes to make his supposed discovery as public as possible, I send it to you for insertion in THE LANCET, if you deem it worthy of a place in that jourual.

Sackville-street, Oct. 1849.

Yours, \&ce.,

Winliam Piout.

Sir,-I do myself the pleasure to communicate to you the important fact (in which you will rejoice)-viz., that the carbonate of soda is a speedy and effectual antidote to the poison of cholera. I have just ascertained this in the treatment of cholera here. I give a teaspoonful of it the moment a patient is brought to me, in a cup of very hot gruel or water. It relieves the pain and burning of stomach and bowels, produces sleep, and restores the pulse and heat in a very short space of time. If it should be vomited, I immediately repeat it with a little laudanum, and a full dose of oil, so as that it shall pass speedily down to the small intestines, where the bulk of the poison appears to rest.

When any portion of the oil is passed in the motion, convalescence will already have commenced, and the patient will presently pass urine, and be then out of all danger. I repeat smaller doses morning and evening for a day or so, if necessary.

When great numbers are attacked at the same time, I used boluses of carbonate of soda, twenty grains; gamboge, five to ten grains; croton oil, two to three grains, or more; opium three grains; soap, twenty grains, washed down with a draught of soda. These boluses and soda, sufficient for great numbers, are easily carried in the pocket: I use nothing else.

I will not at present trouble you with details.

Pray be kind enough to make this letter as public as possible, and $I$ remain, with the greatest respect,
Hydrabad, Deckan,
August, 1849.

$$
\text { Your most obedient servant, }
$$

W. MAXWELL, M.D. Surgeon 3rd Light Cavalry

\section{A NEWLY-PROJECTED MEDICAL BILL.} To the Editor of The LanceT.

Sir,-Knowing the deep interest you take in all matters tending to elevate the medical profession, especially that large though ill-used branch of it-" the general practitioner," I have much pleasure in informing you that a measure is now on the tapis, and which there is little doubt will early next ses. sion of Parliament become law of the land. You well know the mighty stress laid upon a good preliminary education by all parties before the Parliamentary Committee, and that the great bar to this was the five years' clause in the A pothecaries? Act. Now, through the Lord Advocate of Scotland, a Bill is proposed simply repealing this clause, or at all events, curtailing it to two or three years, at the same time declaring all who hold a degree of M.D., together with a surgical diploma from universities and colleges of Great Britain and Ireland, legally entitled to be "general practitioners" throughout her Majesty's dominions: should it be deemed necessary to conciliate the Worshipful Company, it will not be objected to a fee to them by those who so practise in England or Wales; also, that these licentiates, provided they have a surgical diploma, shall have equal scope with the others.

Edinburgh, Sept. 1849 .

I remain, yours faithfully,

J. $M$.

\section{ON THE RATIONALE OF PARTURITION}

\section{To the Editor of The Lanoer.}

$\mathrm{SrR},-$ More than one inquirer is pursuing the investigation of the rationale of parturition experimentally. To Dr. W Tyler Smith I proposed such an inquiry some years ago, and I learn from Dr. Simpson, of Edinburgh, that he has recently undertaken a similar investigation. From these researches the truth will eventually arise, and shed its bright light over an important department of medical science.

For my own part, I compare parturition with the act of deglutition, and with the act of vomiting, and especially I regard it as combining,
1. Reflex Action,
2. Peristaltic Action,
3. Voluntary, and
4. Emotional Influence, with
5. Sphincteric Relaxation.

The question is-how far the first two sources of action can be severed, and measured, as it were, in experiment.

For this latter purpose, an instrument, to be designated a dynamometer, and consisting of a long tube attached to a small sphere of India rubber, and partly filled with mercury, may be employed. By means of this instrument we may reduce the reflex and peristaltic powers, in some measure, to numbers.

But first let me premise, that I propose hereafter to designate the Reflex action and acts by the term Diastaltic. The functions of all the tubal orifices and exits of the animal frame will be thus distinguished from all that is interior, as $d i a$-stal tic, the latter being peri-staltic, a term with which we are all perfectly familiar.

These words are very expressive and well contrasted. Others may be added, if this be deemed necessary, from the same root. An incident nerve may be designated ana-staltic; a reflex nerve, cata-staltic, \&c.

The uterine actions are partly, I should think chiefly, dia staltic, and partly peristaltic; and so of the other acts of in gestion and of expulsion, which are also still further influenced by emotion, modified by volition, and conjoined with sphincteric relaxation.

I formerly performed some interesting experiments on the rabbit and the turtle; which will be found in my volume " On the Diseases and Derangements of the Nervous System," $\$ \$ 323,330$. The cesophagus in the former, and the cloaca in the latter, are both diastaltic and peristaltic. The parturient uterus is doubtless so too. Its action may, we know, be excited through the spinal marrow, even from the mamma; and if detached altogether, and placed on the table, I have no doubt that, like the isolated cesophagus of the rabbit, it would slowly expel its contents. Dr. Tyler Smith actually observed this event, some months ago, in a small bitch which had died without delivery. The abdomen and uterus being opened, the puppies escaped, and the uterus collapsed, or rather contracted, so as almost to disappear.

I need scarely refer to the observations and experiments of M. Brachet, and to the facts on record of post-mortem parturition in the human subject.

In regard to new experiments, this is what $I$ would beg to propose:-

Having placed the animal under the influence of chloroform, and having introduced the dynamometer, first within the abdomen, and afterwards within the uterus, let us gently irritate 